\title{
Prevalence of potentially inappropriate prescribing and prescribing omissions in older Irish adults: findings from The Irish LongituDinal Study on Ageing study (TILDA)
}

\author{
Rose Galvin • Frank Moriarty • Gráinne Cousins • Caitriona Cahir • Nicola Motterlini • \\ Marie Bradley • Carmel M. Hughes • Kathleen Bennett • Susan M. Smith • Tom Fahey • \\ Rose-Anne Kenny
}

Received: 23 October 2013 / Accepted: 17 January 2014 / Published online: 4 February 2014

(C) The Author(s) 2014. This article is published with open access at Springerlink.com

\begin{abstract}
Purpose We sought to estimate the prevalence of potentially inappropriate prescriptions (PIP) and potential prescribing omissions (PPOs) using a subset of the STOPP/START criteria in a population based sample of Irish adults aged $\geq 65$ years using data from The Irish LongituDinal Study on Ageing (TILDA).

Methods A subset of 26 PIP indicators and 10 PPO indicators from the STOPP/START criteria were applied to the TILDA dataset. PIP/PPO prevalence according to individual STOPP/ START criteria and the overall prevalence of PIP/PPO were estimated. The relationship between PIP and PPOs and
\end{abstract}

Electronic supplementary material The online version of this article (doi:10.1007/s00228-014-1651-8) contains supplementary material, which is available to authorized users.

R. Galvin $(\bowtie) \cdot$ F. Moriarty $\cdot$ G. Cousins $\cdot$ N. Motterlini $\cdot$

M. Bradley $\cdot$ S. M. Smith $\cdot$ T. Fahey

HRB Centre for Primary Care Research, Department of General Practice, Royal College of Surgeons in Ireland, 123 St. Stephens Green, Dublin 2, Republic of Ireland

e-mail: rosegalvin@rcsi.ie

M. Bradley $•$ C. M. Hughes

School of Pharmacy, Queen's University Belfast, Belfast, UK

C. Cahir $\cdot$ K. Bennett

Department of Pharmacology and Therapeutics, Trinity College

Dublin, Dublin, Ireland

\section{R.-A. Kenny}

The Irish Longitudinal Study of Ageing, Chemistry Extension,

Trinity College Dublin, Dublin 2, Ireland

G. Cousins

School of Pharmacy, Royal College of Surgeons in Ireland, Dublin, Ireland polypharmacy, age, gender and multimorbidity was examined using logistic regression.

Results The overall prevalence of PIP in the study population $(n=3,454)$ was $14.6 \%$. The most common examples of PIP identified were NSAID with moderate-severe hypertension (200 participants; $5.8 \%$ ) and aspirin with no history of coronary, cerebral, or peripheral vascular symptoms or occlusive event (112 participants; $3.2 \%)$. The overall prevalence of PPOs was $30 \%(n=1,035)$. The most frequent PPO was antihypertensive therapy where systolic blood pressure consistently $>160 \mathrm{mmHg}(n=341,9.9 \%)$, There was a significant association between PIP and PPO and polypharmacy when adjusting for age, sex and multimorbidity (adjusted OR 2.62, $95 \%$ CI 2.05-3.33 for PIP and adjusted OR 1.46, $95 \%$ CI $1.23-1.75$ for prescribing omissions).

Conclusion Our findings indicate prescribing omissions are twice as prevalent as PIP in the elderly using a subset of the STOPP/START criteria as an explicit process measure of potentially inappropriate prescribing and prescribing omissions. Polypharmacy was independently associated with both PPO and PIP. Application of such screening tools to prescribing decisions may reduce unnecessary medication, related adverse events, healthcare utilisation and cost.

Keywords Potentially inappropriate prescribing - Potential prescribing omissions $\cdot$ STOPP $\cdot$ START $\cdot$ Older people

\section{Introduction}

Older adults are a heterogeneous group often presenting with multimorbidity and polypharmacy. Prescribing in older adults is challenging for a number of reasons as any new medication must be considered in the context 
of the physiological effects of the drug, the absorption and excretion of the drug and age-related changes in body composition and physiology [1]. Potentially inappropriate prescribing (PIP) in older people refers to the use of medications that should be generally avoided in such populations and doses or frequencies of administration that should not be exceeded [2, 3]. Medicines that are considered potentially inappropriate lack evidence based indications, are not cost-effective and may pose a higher risk of adverse events including increases in morbidity, adverse drug events, hospitalisation and mortality $[4,5]$. In terms of assessing the appropriateness of prescribing in older people, both implicit and explicit measures of process (providers' actions) and outcome (e.g., adverse drug events) are used [6]. Implicit process measures involve a clinician's judgment of appropriateness for the individual patient based on patient characteristics and published work [7]. Explicit process measures are criterion-based and are derived from published evidence based reviews, expert opinion and/or consensus [8,9].

A comprehensive and explicit process measure of PIP has been developed and validated for use in European countries. The STOPP criteria ( $\underline{\text { Screening }}$ Tool for $\underline{\text { Older }}$ Persons' Prescriptions) are a physiological system based screening tool and comprises 65 clinically significant criteria [10]. The criteria include commonly encountered instances of PIP in older people such as drug-drug and drug-disease interactions, drugs which adversely affect older patients at risk of falls and duplicate drug class prescriptions. In addition, under-prescribing of clinically indicated medications has been identified by an accompanying screening tool known as the START criteria ( Screening Tool to Alert doctors to Right Treatment), comprising $\overline{2} 2$ criteria for potentially appropriate drugs.

There have been few national studies of PIP and PPOs in the general population of older people. In 2007, a subset of the STOPP criteria was applied in a national population study carried out in the Republic of Ireland among people aged $\geq 70$ years, using the Health Services Executive Primary Care Reimbursement Services (HSE-PCRS) pharmacy claims database [6]. This study reported PIP prevalence rates of $36 \%$ in the primary care setting. However, this study was limited in its assessment of PIP as the HSEPCRS database contains no information on diagnosis and so only a subset of the STOPP criteria could be applied. PIP prevalence rates reported may not have adequately reflected all instances of PIP among older people such as drug-disease interactions. Therefore, the overall aim of the current study is to estimate the prevalence of PIP/PPOs, particularly relating to drug-disease interactions, in a nationally representative sample of individuals aged $\geq 65$ years, using a subset of the STOPP/START criteria.

\section{Methods}

Study population

The Irish LongituDinal Study on Ageing (TILDA) is a nationally representative cohort study of people aged 50 years and over and resident in Ireland, charting their health, social and economic circumstances over a 10 -year period. The first wave of the TILDA study has been completed, and we have examined the data from this cross sectional wave in our current study. The STROBE standardised reporting guidelines for cross-sectional studies have been followed to ensure the uniform conduct and reporting of the research. All participants aged $\geq 65$ years are included [11].

\section{Overview of TILDA data collection}

Data for the current study was captured from three methods used to collect data in the TILDA dataset. Participants first completed a computer-assisted personal interview (CAPI) in their own homes. Detailed information on all aspects of the respondents' lives was collected in the CAPI, including economic status, health aspects (physical, mental, service needs and usage) and social participation. Participants were then invited to one of two health centres for a comprehensive health assessment. Health assessments were carried out by qualified and trained research nurses. Cognitive, cardiovascular, gait and balance and vision measurements and tests were taken in the health assessment. Finally, each participant was also requested to complete and return a questionnaire designed to explore certain areas that were considered particularly sensitive for respondents to answer directly to an interviewer (e.g., relationship quality, depression and loneliness). The names of medications that participants were taking at the time of the CAPI, including over-the-counter medications, were coded in the TILDA dataset using the World Health Organization (WHO) Anatomical Therapeutic Chemical (ATC) classification system. No information was collected on strength, quantity, duration or cost of prescription.

\section{Application of the STOPP/START criteria}

Inappropriate drug-drug and drug-disease combinations according to the STOPP criteria were identified using information on medications and diagnoses (e.g., hypertension, chronic obstructive pulmonary disease, glaucoma) from the TILDA dataset. In the absence of diagnosis information, prescription drugs for the treatment of certain conditions were used as proxies (e.g., gout, epilepsy). Medications documented by the field-researchers at the CAPI interview were recorded in the TILDA dataset using the WHO ATC classification system. A similar approach was used to identify PPOs. Twenty-six STOPP criteria and ten START criteria were applied to the 
TILDA database. Table 1 contains details of the criteria applied per physiological system. Reasons for exclusion of criteria are contained in Supplemental Table 1.

\section{Statistical analysis}

Data analyses were performed using Stata version 12 (StataCorp, College Station, Tx, USA) and statistical significance at $p<0.05$ was assumed. Descriptive statistics were used to summarise the population. The overall prevalence of PIP/ PPO and the prevalence per individual STOPP/START criteria were calculated as a proportion of all eligible persons $\geq 65$ years. The prevalence of STOPP/START criteria was also calculated as a proportion of the overall disease or drug prevalence (e.g., beta-blocker with COPD as a proportion of COPD prevalence or two concurrent opiates as a proportion of opiate prevalence). Participants were further classified by gender and age group ( $65-74$ years, $\geq 75$ years). Other explanatory variables examined included polypharmacy, defined as concurrent use of five or more medications [12] and number of chronic conditions (none versus 1, 2, 3 or more). Univariate analyses confirmed that explanatory variables (polypharmacy, age, gender and multimorbidity) were significantly associated with PIP/PPOs. The association among any PIP/PPO (versus none) and age, gender, polypharmacy and multimorbidity was assessed using logistic regression and presented as adjusted odds ratios with $95 \%$ confidence intervals.

Table 1 STOPP/START criteria applied to the TILDA dataset

\begin{tabular}{lll}
\hline Physiological system & $\begin{array}{l}\text { Number of } \\
\text { criteria included }\end{array}$ & $\begin{array}{l}\text { Percentage of } \\
\text { criteria included }\end{array}$ \\
\hline STOPP criteria & & \\
Cardiovascular system & $8 / 17$ & $47.1 \%$ \\
CNS \& psychotropic drugs & $4 / 13$ & $30.8 \%$ \\
Gastrointestinal system & $1 / 5$ & $20 \%$ \\
Respiratory system & $2 / 3$ & $66.7 \%$ \\
Musculoskeletal system & $4 / 8$ & $50 \%$ \\
Urogenital system & $2 / 6$ & $33.3 \%$ \\
Endocrine system & $1 / 4$ & $25 \%$ \\
Drugs that adversely affect & $3 / 5$ & $60 \%$ \\
those prone to falls & $0 / 3$ & $0 \%$ \\
Analgesic drugs & $1 / 1$ & $100 \%$ \\
Duplicate drug classes & & \\
START criteria & $5 / 8$ & $62.5 \%$ \\
Cardiovascular system & $0 / 3$ & $0 \%$ \\
Respiratory system & $2 / 2$ & $100 \%$ \\
Central nervous system & $0 / 2$ & $0 \%$ \\
Gastrointestinal system & $0 / 3$ & $0 \%$ \\
Musculoskeletal system & $3 / 4$ & $75 \%$ \\
Endocrine system & & \\
\hline
\end{tabular}

\section{Results}

Population descriptive statistics

A total of 3,507 people $\geq 65$ years in Ireland were identified from the TILDA database of which 1,842 (52.5\%) were female and 1,665 (47.5\%) were male. Almost two thirds of individuals were aged between 65 and 74 years $(n=2,163)$. The remaining $38 \%(n=1,344)$ were aged $\geq 75$ years. Fiftythree participants reported missing medication data; theref,ore the STOPP/START criteria were applied to 3,454 participants.

\section{Overall prevalence of PIP and PPOs}

The overall prevalence of PIP was $14.6 \%(n=504)$ considering all 26 STOPP criteria. Almost $13 \%$ of the population $(n=$ 433) were prescribed one potentially inappropriate medication, $60(1.7 \%)$ were prescribed two and $11(0.3 \%)$ were prescribed three or more. The overall prevalence of PPOs was $30 \%(n=1,035)$ considering the ten included START criteria. Eight hundred and four individuals $(23.3 \%)$ presented with one PPO, 188 (5.4\%) with two and 43 (1.2\%) with three or more PPOs.

Prevalence of PIP according to individual STOPP criteria

Table 2 presents the prevalence of each of the individual STOPP criteria by physiological system. Prescription of a non-steroidal anti-inflammatory drug (NSAID) with moderate-severe hypertension was the most frequent potentially inappropriate drug $(n=200,5.8 \%)$. The second most frequent PIP was aspirin with no history of coronary, cerebral, or peripheral vascular symptoms or occlusive event $(n=112$, $3.2 \%$ ), followed by benzodiazepines in those prone to falls $(n=40,1.2 \%)$ and aspirin with a past history of peptic ulcer disease without a histamine $\mathrm{H}_{2}$ receptor antagonist or proton pump inhibitor $(n=38,1.1 \%)$. Angiotensin Converting Enzyme (ACE) inhibitors $(n=23,0.7 \%)$ and NSAIDs $(n=21$, $0.6 \%$ ) were the most frequently prescribed duplicate drugs (Table 2).

Prevalence of PPOs according to individual START criteria

Table 3 presents the prevalence of each of the individual START criteria by physiological system. The most frequent PPO was antihypertensive therapy where systolic blood pressure consistently $>160 \mathrm{mmHg}(n=341,9.9 \%)$, followed by the omission of warfarin in the presence of chronic atrial fibrillation $(n=270,7.8 \%)$ and statin therapy in diabetes mellitus if one or more co-existing major cardiovascular risk factors were present $(n=235,6.8 \%)$. 
Table 2 STOPP criteria applied to TILDA data for all those aged $\geq 65$ years in Ireland in 2010

STOPP criteria description
Potentially inappropriate prescription (n)
Potentially inappropriate prescription (\%)
Proportionate prescribing per indication $(\%)^{\mathrm{a}}$

Cardiovascular system

Loop diuretic as first line-monotherapy for hypertension (safer, more effective alternatives available)

Thiazide diuretic with a history of gout (may exacerbate gout)

Beta-blocker with COPD (risk of increased bronchospasm)

Beta-blocker with verapamil (risk of symptomatic heart block)

Aspirin and warfarin without histamine $\mathrm{H}_{2}$ receptor antagonist (except cimetidine) or proton pump inhibitor (high risk of gastrointestinal bleeding)

Dipyridamole as monotherapy for cardiovascular secondary prevention (no evidence of efficacy)

Aspirin with a past history of peptic ulcer disease without histamine $\mathrm{H}_{2}$ receptor antagonist or Proton Pump Inhibitor (risk of bleeding)

Aspirin with no history of coronary, cerebral, or peripheral vascular symptoms or occlusive event (not indicated)

Central nervous system and psychotropic drugs

TCA and glaucoma (exacerbate glaucoma)

TCA and opiate or calcium channel blockers (risk of severe constipation)

Phenothiazines in patients with epilepsy (may lower seizure threshold)

Anticholinergics to treat extra-pyramidal side-effects of neuroleptic medications (risk of anticholinergic toxicity)

Gastrointestinal system

Prochlorperazine or metoclopramide with parkinsonism (risk of exacerbating parkinsonism)

Respiratory system

Theophylline as monotherapy for COPD (safer, more effective alternative: risk of adverse effects due to narrow therapeutic index)

Nebulised ipratropium with glaucoma (exacerbate glaucoma)

Musculoskeletal system

NSAID with history of peptic ulcer disease or gastrointestinal bleeding, unless with concurrent histamine $\mathrm{H}_{2}$ receptor antagonist, PPI or misoprostol (risk of peptic ulcer relapse)

NSAID with moderate-severe hypertension (moderate: 160/100-179/109 mmHg; severe: $\geq 180 / 110 \mathrm{mmHg}$ ) (risk of exacerbation of hypertension)

NSAID with heart failure (risk of exacerbation of heart failure)

Warfarin and NSAID (risk of gastrointestinal bleeding)

Urogenital system

Antimuscarinic drugs with chronic glaucoma ( $>3$ months) (risk of acute exacerbation of glaucoma) $^{\mathrm{b}}$

Alpha-blockers in males with frequent incontinence i.e., one or more episodes of incontinence daily (risk of urinary frequency and worsening of incontinence) ${ }^{\mathrm{c}}$

Endocrine system

Glibenclamide or chlorpropamide with type 2 diabetes mellitus (risk of prolonged hypoglycemia)

Drugs that adversely affect those prone to falls $(\geq 1$ fall in past 3 months)

Benzodiazepines (sedative, may cause reduced sensorium, impair balance) ${ }^{\mathrm{d}} \quad 40$

Neuroleptic drugs (may cause gait dyspraxia, Parkinsonism) ${ }^{\mathrm{d}}$

First generation antihistamines (sedative, may impair sensorium) ${ }^{\mathrm{d}}$

Duplicate drug class prescription (optimization of monotherapy within a single drug class)

Two concurrent opiates

Two concurrent NSAIDs

Two concurrent SSRIs
9

3

0.26

0.09

3.80

0.90

18.02

0.52

1.00

0.38

0.61 
Table 2 (continued)

\begin{tabular}{llll}
\hline STOPP criteria description & $\begin{array}{l}\text { Potentially } \\
\text { inappropriate } \\
\text { prescription (n) }\end{array}$ & $\begin{array}{l}\text { Potentially } \\
\text { inappropriate } \\
\text { prescription (\%) }\end{array}$ & $\begin{array}{l}\text { Proportionate } \\
\text { prescribing per } \\
\text { indication (\%) }\end{array}$ \\
\hline Two concurrent antidepressants & 2 & 0.06 & 3.03 \\
Two concurrent loop diuretics & 0 & - & - \\
Two concurrent ACE inhibitors & 23 & 0.67 & 2.28 \\
\hline
\end{tabular}

COPD chronic obstructive pulmonary disease, TCA tricyclic antidepressant, NSAID non-steroidal anti-inflammatory drug, SSRI selective serotonin reuptake inhibitor, $A C E$ inhibitors angiotensin converting enzyme inhibitors and Angiotensin receptor blockers

${ }^{\text {a }}$ Proportionate prescribing per indication, e.g., prevalence of STOPP criteria as a proportion of the overall disease or drug prevalence, e.g.,Beta-blocker with COPD as a proportion of COPD prevalence, two concurrent opiates as a proportion of opiates prevalence

${ }^{\mathrm{b}} 8(0.23 \%)$ missing data for chronic glaucoma variable

${ }^{\mathrm{c}}$ Proportion of male participants only, $5(0.30 \%)$ missing data for urinary incontinence variable

${ }^{\mathrm{d}} 1(0.03 \%)$ missing data for falls in past year variable

Factors associated with PIP

Any PIP was more likely in people $\geq 75$ years than those 65 74 years when adjusting for gender (OR 1.41, $95 \%$ CI 1.161.70). This association remained significant after additionally adjusting for polypharmacy (age $\geq 75$ years vs. $65-74$ years, OR $1.22,95 \%$ CI 1.00-1.50). There was no association between PIP and gender when adjusting for age (F vs. M, OR 1.05, $95 \%$ CI 0.87-1.26). Supplemental Table 2 presents the association between gender and age and PIP by individual
STOPP criteria. There was a strong association between any PIP and polypharmacy when accounting for age and gender (OR 3.13, $95 \%$ CI 2.53-3.87), and this association remained significant when additionally adjusting for the presence of chronic conditions (OR 2.62, 95 \% CI 2.05-3.33).

\section{Factors associated with PPOs}

There was no difference in the likelihood of a PPO in those $\geq 75$ years when compared to those 65-74 years when

Table 3 START (Screening Tool to Alert doctors to Right Treatment) criteria applied to TILDA data for all those aged $\geq 65$ years in Ireland in 2010

\begin{tabular}{|c|c|c|c|}
\hline START criteria description & $\begin{array}{l}\text { Potential } \\
\text { prescribing } \\
\text { omissions (n) }\end{array}$ & $\begin{array}{l}\text { Potential } \\
\text { prescribing } \\
\text { omissions }(\%)\end{array}$ & $\begin{array}{l}\text { Proportionate prescribing } \\
\text { omission per indication }(\%)^{*}\end{array}$ \\
\hline \multicolumn{4}{|l|}{ Cardiovascular system } \\
\hline Warfarin in the presence of chronic atrial fibrillation & 270 & 7.82 & 75.00 \\
\hline Antihypertensive therapy where systolic blood pressure consistently $>160 \mathrm{mmHg} \dagger$ & 341 & 9.87 & 18.62 \\
\hline Angiotensin Converting Enzyme (ACE) inhibitor with chronic heart failure & 23 & 0.67 & 42.59 \\
\hline ACE inhibitor following acute myocardial infarction & 126 & 3.65 & 47.19 \\
\hline Beta-blocker with chronic stable angina & 151 & 4.37 & 45.21 \\
\hline \multicolumn{4}{|l|}{ Central nervous system } \\
\hline $\begin{array}{l}\text { L-DOPA in idiopathic Parkinson's disease with definite functional } \\
\text { impairment and resultant disability }\end{array}$ & 3 & 0.09 & 17.65 \\
\hline $\begin{array}{l}\text { Antidepressant drug in the presence of moderate-severe depressive } \\
\text { symptoms lasting at least } 3 \text { months } \S\end{array}$ & 44 & 1.30 & 70.97 \\
\hline \multicolumn{4}{|l|}{ Endocrine system } \\
\hline $\begin{array}{l}\text { ACE inhibitor or Angiotensin Receptor Blocker in diabetes with } \\
\text { nephropathy, i.e., overt urinalysis proteinuria }\end{array}$ & 13 & 0.38 & 44.83 \\
\hline $\begin{array}{l}\text { Antiplatelet therapy in diabetes mellitus if one or more co-existing } \\
\text { major cardiovascular risk factor present (hypertension, } \\
\text { hypercholesterolaemia, smoking history) }\end{array}$ & 110 & 3.18 & 35.48 \\
\hline $\begin{array}{l}\text { Statin therapy in diabetes mellitus if one or more co-existing major } \\
\text { cardiovascular risk factor present }\end{array}$ & 235 & 6.80 & 75.81 \\
\hline
\end{tabular}

* Proportionate prescribing omission per indication, e.g. prevalence of PPO as a proportion of the overall disease, e.g. no warfarin with chronic atrial fibrillation as a proportion of chronic atrial fibrillation prevalence

$\S 70(2.03 \%)$ missing data for depressive symptoms variable

$\dagger 1,119$ (32.40\%) missing data for blood pressure variable 
adjusting for gender (OR 1.09, $95 \%$ CI 0.92-1.28). However, omissions were significantly more likely in males when compared to females when adjusting for age (OR $0.73,95 \% \mathrm{CI}$ $0.63-0.85)$. The association between PPOs and gender remained significant after also adjusting for polypharmacy (OR 0.71, $95 \%$ CI 0.61-0.83). Supplemental Table 3 presents the association between gender and age and PPOs by individual START criteria. There was a significant association between PPOs and polypharmacy when adjusting for age and gender (OR 2.28, $95 \%$ CI 1.93-2.69) and this association also remained significant when also adjusting for number of chronic conditions (OR 1.46, $95 \%$ CI 1.23-1.75).

\section{Discussion}

\section{Statement of principal findings}

This current study found that almost $15 \%$ of people aged $\geq 65$ years in the Republic of Ireland received at least one PIP, according to a subset of 26 STOPP criteria. PIP was more likely in older adults ( $\geq 75$ years) when adjusting for gender and polypharmacy. The overall prevalence of potential prescribing omissions was $30 \%$ considering the ten included START criteria. Omissions were significantly more likely in males when compared to females when adjusting for age and polypharmacy. There was a significant association between PIP/PPOs and polypharmacy when adjusting for age, gender and multimorbidity.

\section{Results in the context of current literature}

This study presents a snapshot of the prevalence of PIP in a nationally representative population of people aged $\geq 65$ years using data from the first wave of TILDA. Previous research is limited by having focused on specific groups in particular clinical settings as well as having measured PIP using Beers' criteria derived in the US [13-16]. A limited number of studies of PIP in the general population of older people exist. In a previous Irish study using data from the HSE-PCRS, an overall prevalence of $36 \%$ in patients $\geq 70$ years was reported using a subset of 30 STOPP criteria [6]. Similar results were reported in a study of the Enhanced Prescribing Database (EPD) in Northern Ireland where the overall prevalence of PIP was $34 \%$ [5]. However, the differences between the TILDA database and these prescription reimbursement databases make comparisons more difficult. The lack of clinical information relating to diagnoses (e.g., hypertension, CVA) in the EPD and PRCS databases and the lack of information on drug duration, dose or frequency of administration of prescriptions in the TILDA dataset mean that only a limited number of the STOPP criteria applied in the three studies are similar. In the EPD and PCRS databases, the most prevalent PIP drugs were proton pump inhibitors (PPIs) at maximum therapeutic dosage for $>8$ weeks, followed by NSAIDs for $>3$ months and long-acting benzodiazepines for $>1$ month [5, 6]. These instances of PIP were not captured in TILDA. Similarly, the use of a NSAID with moderate-severe hypertension was the most prevalent PIP in TILDA, and this could not be assessed in the prescription databases due to a lack of clinical information. Of the 19 STOPP criteria that were the same in PCRS and TILDA, the prevalence of PIPs are broadly similar, but low in both datasets $(<2.5 \%)$.

The significant association between PIP and polypharmacy is consistent with findings reported in previous studies $[5,6$, 17], even when age, gender and multimorbidity is accounted for [18]. While it is well recognised that the use of multiple medications in the older adults is associated with increased risk of adverse events such as adverse drug events in patients taking NSAIDs (e.g., gastrointestinal haemorrhage) and increased incidence of falls and cognitive impairment in those taking sedative-hypnotic drugs, these medications are routinely among the most common PIPs [3, 6, 19], as was the case in our study. We also found an association between advancing age and PIP after adjusting for polypharmacy, similar to a previous primary care based study [20]. We reported no association between PIP and gender after adjustments for age. These findings are contrary to previous studies reporting that women are more likely to receive a PIP when compared with men $[6,17,21]$.

Our overall findings relating to the prevalence of PPOs are similar to previous studies that have used the START criteria in a primary care setting [20]. Other studies have reported higher prevalence rates of omissions among an older hospital inpatient population $[22,23]$. However, older patients admitted to hospital are sicker and frailer than those in the community so the findings need to be considered in the context of the population of interest. The cardiovascular system accounted for the majority of PPOs in keeping with previous reports [20]. Our study demonstrated that polypharmacy is associated with the under-prescribing of indicated medicines even when adjusting for age, gender and co-morbid conditions. Similar findings have been reported in other studies, particularly those with co-morbid conditions [24, 25]. However, these findings warrant further investigation.

We also noted that over $70 \%$ of study participants who reported moderate-severe depressive symptoms were not prescribed an antidepressant drug. Depressive symptoms were assessed in TILDA using the Center for Epidemiologic Studies Depression Scale (CES-D) [26]. We used a cut off score of $\geq 27$ points on the CES-D scale as a proxy for diagnosis of 'moderate-severe' depressive symptoms. This interpretation is the most conservative estimate reported in the literature [27]; therefore, our findings need to be considered in the context of these shortcomings. It may be that other management approaches were adopted in the treatment of depressive symptoms such as cognitive behavioural therapy [28]. 
Clinical and policy implications

The term 'potentially' is used as there is often limited evidence to support the inclusion of particular medications in lists of drugs to avoid in particular populations. However, their use or lack thereof may be indicated and appropriate in certain circumstances considering the clinical presentation of the patient and the balance of the risk and benefits [5]. Clinical vigilance and quality efforts should place particular focus on medication appropriateness, both with respect to under-prescribing and over-prescribing. Reducing PIP in older people will require implementation of more robust methods of medication reviews to routinely assess drug effectiveness, dosage, duration, interactions and adverse effects [29, 30].

The updated NICE guidelines (2013) on falls assessment and prevention in older people highlight the importance of a medication review in older people at risk of falls who present to healthcare professionals. There is a lack of experimental research on the use of the STOPP criteria as a tool to reduce the incidence of adverse drug events (ADEs) such as falls in older people. However, a recent randomised controlled trial demonstrated that the application of the STOPP criteria significantly improved medication appropriateness when compared with the usual pharmaceutical care in 400 hospital inpatients [3]. The study was not powered to detect a reduction in secondary outcomes such as ADEs but further research is warranted to examine the role of medicines reviews based on the STOPP criteria as an intervention tool to attenuate ADE incidence. In addition, reducing the number of drugs used by older people may serve to reduce the risk of falls and the associated direct and indirect costs [29].

Strengths and weaknesses of the study

The TILDA database contains data on morbidities ( $>99.5 \%$ complete) that enable the assessment of PIP in the context of a given diagnosis (e.g., hypertension, cardiovascular conditions). These data also facilitate the assessment and measurement of errors of omission, i.e., the lack of use of appropriate drugs when needed. However, it was only possible to apply a subset of the STOPP/START criteria to the database, as described in Table 1, due to a lack of information on drug strength, dose and duration of prescriptions. This may have led to an underestimate of the true prevalence of PIP/PPO among older people in TILDA. In addition, the validity of the original criteria is diminished by only applying a subset and the comparability to other population based studies is limited due to the differences in criteria applied.

The cost of PIP was not considered in the present study, but a previous study estimated the cost of PIP to be in excess of $€ 45$ million using a subset of 30 STOPP criteria [6]. Further research is planned to link the TILDA dataset to the national prescribing database (HSE-PCRS) to facilitate the application of 19 additional STOPP criteria and to estimate the cost of PIP using these linked datasets. In addition, there is a need to compare these findings with other European population based studies. The association between explicit process measures of PIP and prescribing omissions, and health outcomes (morbidity and mortality) and healthcare utilisation also requires further investigation to facilitate the development and testing of appropriate interventions to reduce PIP and PPOs in older adults. In the Irish context, this can be completed using future waves of the TILDA data.

\section{Conclusion}

Our findings indicate PIP and PPOs are prevalent in the elderly using a subset of the STOPP/START criteria as an explicit process measure of potentially inappropriate prescribing and PPOs. Application of such screening tools to prescribing decisions may reduce unnecessary medication, related adverse events, healthcare utilisation and cost.

Competing interests The authors declare that they have no competing interests.

Contribution of authors All authors were involved in the study conception and design. RG and GC identified the eligible STOPP/START criteria. NM, CC and FM completed the statistical analysis. RG, GC and $\mathrm{MB}$ interpreted the data and drafted the paper. TF, RK, KB, CH and SS provided methodological and content expertise during the manuscript preparation and critically revised the draft manuscript. All authors read and approved the final manuscript.

Funding The TILDA study was supported by Department of Health and Children, The Atlantic Philanthropies and Irish Life. This work was also supported by the Health Research Board (HRB) of Ireland through the HRB Centre for Primary Care Research under Grant HRC/2007/1.

Open Access This article is distributed under the terms of the Creative Commons Attribution License which permits any use, distribution, and reproduction in any medium, provided the original author(s) and the source are credited.

\section{References}

1. Gallagher P, Barry P, O’Mahony D (2007) Inappropriate prescribing in the elderly. J Clin Pharm Ther 32:113-121

2. O'Mahony D, Gallagher PF (2008) Inappropriate prescribing in the older population: need for new criteria. Age Ageing 37:138-141

3. Gallagher PF, O'Connor MN, O'Mahony D (2011) Prevention of potentially inappropriate prescribing for elderly patients: a randomized controlled trial using STOPP/START criteria. Clin Pharmacol Ther 89:845-854

4. Gallagher P, O'Mahony D (2008) STOPP (Screening Tool of Older Persons' potentially inappropriate Prescriptions): application to acutely ill elderly patients and comparison with Beers' criteria. Age Ageing 37:673-679 
5. Bradley MC, Fahey T, Cahir C, Bennett K, O’Reilly D, Parsons C, Hughes CM (2012) Potentially inappropriate prescribing and cost outcomes for older people: a cross-sectional study using the Northern Ireland enhanced prescribing database. Eur J Clin Pharmacol 68: $1425-1433$

6. Cahir C, Fahey T, Teeling M, Teljeur C, Feely J, Bennett K (2010) Potentially inappropriate prescribing and cost outcomes for older people: a national population study. Br J Clin Pharmacol 69:543-552

7. Samsa GP, Hanlon JT, Schmader KE, Weinberger M, Clipp EC, Uttech KM, Lewis IK, Landsman PB, Cohen HJ (1994) A summated score for the medication appropriateness index: development and assessment of clinimetric properties including content validity. J Clin Epidemiol 47:891-896

8. Fialova D, Onder G (2009) Medication errors in elderly people: contributing factors and future perspectives. Br J Clin Pharmacol 67:641-645

9. Beers MH (1997) Explicit criteria for determining potentially inappropriate medication use by the elderly. An update. Arch Intern Med 157:1531-1536

10. Gallagher P, Ryan C, Byrne S, Kennedy J, O’Mahony D (2008) STOPP (screening tool of older person's prescriptions) and START (screening tool to alert doctors to right treatment). Consensus validation. Int J Clin Pharmacol Ther 46:72-83

11. Von Elm E, Altman DG, Egger M, Pocock SJ, Gotzsche PC, Vandenbroucke JP (2007) The STrengthening the Reporting of OBservational studies in Epidemiology (STROBE) statement: guidelines for reporting observational studies. PLoS Med 4:e296

12. Viktil K, Blix H, Moger T, Reikvam A (2007) Polypharmacy as commonly defined is an indicator of limited value in the assessment of drug-related problems. Br J Clin Pharmacol 63:187-195

13. Wahab MS, Nyfort-Hansen K, Kowalski SR (2012) Inappropriate prescribing in hospitalised Australian elderly as determined by the STOPP criteria. Int J Clin Pharm 34:855-862

14. Hamilton H, Gallagher P, Ryan C, Byrne S, O’Mahony D (2011) Potentially inappropriate medications defined by STOPP criteria and the risk of adverse drug events in older hospitalized patients. Arch Intern Med 171:1013-1019

15. Ryan C, O'Mahony D, Kennedy J, Weedle P, Cottrell E, Heffernan M, O'Mahony B, Byrne S (2012) Potentially inappropriate prescribing in older residents in Irish nursing homes. Age Ageing 42:116-120

16. Van der Hooft CS, Jong GW, Dieleman JP, Verhamme KM, van der Cammen TJ, Stricker BH, Sturkenboom MC (2005) Inappropriate drug prescribing in older adults: the updated 2002 Beers criteria - a population-based cohort study. Br J Clin Pharmacol 60:137-144

17. Carey IM, De Wilde S, Harris T, Victor C, Richards N, Hilton SR, Cook DG (2008) What factors predict potentially inappropriate primary care prescribing in older people? Analysis of uk primary care patient record database. Drugs Aging 25:693-706

18. Nobili A, Marengoni A, Tettamanti M, Salerno F, Pasina L, Franchi C, Iorio A, Marcucci M, Corrao S, Licata G, Mannucci PM (2011) Association between clusters of diseases and polypharmacy in hospitalized elderly patients: results from the reposi study. Eur J Intern Med 22:597-602

19. Ryan C, O'Mahony D, Byrne S (2009) Application of STOPP and START criteria: interrater reliability among pharmacists. Ann Pharmacother 43:1239-1244

20. Ryan C, O’Mahony D, Kennedy J, Weedle P, Byrne S (2009) Potentially inappropriate prescribing in an Irish elderly population in primary care. Br J Clin Pharmacol 68:936-947

21. Liu CL, Peng LN, Chen YT, Lin MH, Liu LK, Chen LK (2012) Potentially inappropriate prescribing (IP) for elderly medical inpatients in Taiwan: a hospital-based study. Arch Gerontol Geriatr 55: $148-151$

22. Barry P, Gallagher P, Ryan C, O'Mahoney D (2007) START (Screening Tool to Alert doctors to the Right Treatment) - an evidence-based screening tool to detect prescribing omissions in elderly patients. Age Ageing 36:632-638

23. Dalleur O, Spinewine A, Henrard S, Losseau C, Speybroeck N, Boland B (2012) Inappropriate prescribing and related hospital admissions in frail older persons according to the STOPP and START criteria. Drugs Aging 29:829-837

24. Kuijpers M, van Marum RJ, Egberts A, Jansen P, OLDY study group (2007) Relationship between polypharmacy and underprescribing. $\mathrm{Br}$ J Clin Pharmacol 65:130-133

25. Steinman M, Landefeld S, Rosenthal G, Berthenthal D, Sen S, Kaboli $\mathrm{P}$ (2006) Polypharmacy and prescribing quality in older people. J Am Geriatr Soc 54:1516-1523

26. Radloff L (1977) The CES-D scale: a self-report depression scale for research in the general population. Appl Psychol Meas 1:385-401

27. Blumenthal J, Lett H, Babyak M, White W, Smith P, Mark D, Jones R, Mathew J, Newman M, NORG Investigators (2003) Depression as a risk factor for mortality after coronary artery bypass surgery. Lancet 362:604-609

28. DeRubeis R, Hollon S, Amsterdam J, Shelton R, Young P, Salomon R, O'Reardon J, Lovett M, Gladis M, Brown L, Gallop R (2005) Cognitive therapy vs medications in the treatment of moderate to severe depression. Arch Gen Psychiatry 62:409-416

29. McMahon G, Cahir C, Kenny R, Bennett K (2013) Inappropriate prescribing in older fallers presenting to an Irish emergency department. Age Ageing. doi:10.1093/ageing/aft114

30. Steinman M, Handler S, Gurwitz J, Schiff G, Covinsky K (2011) Beyond the prescription: medication monitoring and adverse drug events in older adults. J Am Geriatr Soc 59:1513-1520 